\title{
Child and adolescent psychiatric disorders in general practice R. G. Jezzard
}

It has been known for some time that the majority of adult mental health problems within any community are seen within general practice, making up a fifth to a quarter of the general practitioner's workload (Shepherd \& Clare, 1981). Most patients are not referred to specialist services. It is therefore the primary care team and not the psychiatric specialist who assumes the major burden of responsibility for the care of mental disorder. Hence, more attempts are now being made to improve the quality of psychiatric care within general practice. Most of the research on mental health morbidity within primary care has been in the field of adult mental health and it is only relatively recently that equivalent information on child mental health morbidity has become available. In parallel, an increasing number of studies examining the effectiveness of interventions in primary care have been undertaken and different models of mental health service provision within this context have been described (Strathdee \& Williams, 1984). There are few evaluated studies of interventions in primary care focused on child mental health. Most guidance for the development of such services has to be obtained from the adult mental health literature, or from work with other types of childhood disorder, often in non-general practice settings.

\section{Changes in service provision}

The combination of primary care focused research and a number of policy changes (e.g. Community Care legislation) has led to increased expectations that general practitioners and other members of the primary care team should play a larger role in the care of their patients with mental health problems. There is also an increased awareness of hitherto poorly met or unrecognised need, particularly for those with less severe mental health problems. Faced with these considerable challenges, primary care and specialist and hospital-based services are having to redefine their respective roles. General practitioners are now playing an increasingly important part in determining the shape and type of services available to their patients within both the primary and the secondary care system.

Changes have also occurred within the field of child care generally. Social services departments have had to better define their priorities and the reorganisation of education services has led to alterations in the specialist service provision in both ordinary and special schools and within the special needs sector. All services are expected to target their resources more effectively. These changes can be set against a reported increase in waiting lists (66\%) in child psychiatric clinics (Kurtz et al, 1994). The majority of these services have found that referral rates have substantially increased and secondary services have found it increasingly hard to meet the demand. Even those specialist services which appear to be well targeted fail to reach a large number of children in need (Evans \& Brown, 1993). This is despite the fact that the children themselves are usually known to teachers and are often regular attenders at general practice. This brings into focus once again the potential role of the primary care team.

\section{Child care, the family and general practice}

Gray (1994), in a recently published book on psychiatry in general practice points out that

"Most of the recent theory on family relationships has been derived more from social work and child psychiatry than from general practice, which has contributed little, considering the unique perspective on families which family doctors have."

The opportunities for developing an understanding of family problems, and children's

Dr Bob Jezzard is a Consultant Child and Adolescent Psychiatrist based at the Bloomfield Centre, Guy's Hospital, London. 
problems in particular are, however, considerable and many family doctors are in a position to have a unique intergenerational perspective on children's development. While the contribution to theory may be surprisingly little, the GP's role in relation to the care of children is extremely significant and has increased to include formal responsibilities for all aspects of child health surveillance.

Work with children constitutes about $25 \%$ of the work of a GP. An average sized practice may therefore expect to look after about 500 children leading to approximately 1400 child consultations per year (Peter, 1993). General practitioners have most contact with younger children and children under 5 see their doctor up to eight times per year on average. Given that over $90 \%$ of GPs provide antenatal care and that children are registered with their mothers more than $90 \%$ of the time, they have a crucial role in child care and can intervene at an early stage in the development of problems. The early identification and treatment of postnatal depression, for instance, will not only benefit the mother but also the child. With this kind of illustration in mind, Williams (1993) called for an increased awareness among GPs of the emotional needs of children and for the improved recognition and treatment of those with psychiatric disorder.

The linkage between child health and family mental health issues was demonstrated in Shepherd's study (1981). Children of neurotic women experienced more illness than did controls and had a higher rate of emotional disturbance. The study further suggested that the presence of emotional disturbance in the children was related to the mental health of the mother more than the father. It follows that an important part of child health surveillance may be enquiring about and systematically thinking about the emerging and developing parent-child relationship (Gray, 1994). However, GPs are not always the first professionals that parents tend to consult about disturbed behaviour in their children. A study modelling the referral pathway to mental

Box 1. Advantages of general practice settings for the identification and treatment of child mental health problems

GPs can have an intergenerational perspective

GPs have regular opportunities to observe child/parent relationships

General practice is seen to be less stigmatising

Contact levels present many opportunities for intervention
Box 2. Features of general practice relevant to family relationships and children (from Gray, 1994)

Two-thirds of all family planning is offered through general practice

GPs meet the male partner in four out of five family units

Over $90 \%$ of GPs provide antenatal care

Forty-two per cent of NHS patients have been looked after by their GP for 20 or more years

Children are registered with their mother's family doctor over $95 \%$ of the time

Children under five see a GP as many as eight times a year

Babies are seen every six to seven weeks on average

Families are seen interacting at times of stress

health services for children suggests that in the case of 3-year-olds parents usually first go to health visitors; for 10-year-olds they go to teachers (Wolpert \& Fredman, 1994). Nonetheless, the GP's contact with children and families presents many opportunities for intervention.

\section{The mental health morbidity of children within general practice}

It has been estimated that approximately $10-20 \%$ of children within the general population at any one time has a mental health problem. However it is only about $10 \%$ of these that receive help from specialist services (Rutter et al, 1970), which suggests that a very high percentage of children with mental health problems go unrecognised or that they are dealt with in other settings. One of the first studies in this country to assess child mental health morbidity within general practice showed that only $3.5 \%$ of all consultations were about 'pure' psychological problems (Bailey et al, 1978). However, if those with physical symptoms and associated psychological factors were included, together with psychological problems present in mother or family, a figure of $48.4 \%$ of all consultations had a psychological or psychosocial component. Similarly, Garralda \& Bailey (1986) found that $23 \%$ of children aged 7-12 attending a 
general practice had psychiatric disturbance although only $8 \%$ of them presented with emotional or behavioural problems as the primary complaint; most presented with somatic symptoms. Although anxiety was identified in a number, only three children in their sample presented with this. In this study there was an excess of girls, a higher percentage of emotional disorders than would be prevalent in the general population, and an association with psychosocial disadvantage.

Psychiatric morbidity is also more common among frequent attenders in general practice, $29 \%$ as opposed to $9 \%$ (Bowman \& Garralda, 1993), and is common amongst those who attend for physical problems only. That as many as $40 \%$ of children in the population with psychiatric disorders are frequent attenders at general practice indicates how important primary care settings are for the identification of problems, especially emotional disorders. The decisions about whether to present these mental health problems to the doctor are inevitably very much dependent on the views and attitudes of the family.

While psychiatric disorders can be commonly found in children attending general practices, they are rarely recorded, especially in young children. However, rate of recording increases through adolescence into adulthood (Smeeton, 1987), and a diagnosis made in adolescence is associated with an increased likelihood of psychiatric disorder being recorded in early adulthood (Smeeton, 1992). Hence, early recognition and treatment of these problems is necessary and, as Smeeton indicates, should be carried out in primary care where possible.

\section{Common problems}

There are numerous occasions when GPs will be called upon to offer advice on common problems with a mental health component. Minor problems can cause considerable distress not only in the child but also in the parents, particularly if there are coexisting family tensions. The secondary consequences of unresolved difficulties can be considerable. Nocturnal enuresis, for instance, if left untreated and if it persists into middle or late childhood may have considerable effects upon the child's self-esteem and peer relationships. Many problems can be nipped in the bud by early behavioural advice which not only may give a practical solution to solving the problem but will also lead to the reduction of parental anxiety (Horne, 1992). Sleep problems, temper tantrums and feeding problems are very common in infants and young children (Markus et al, 1991). If identified and managed appropriately, the development of persistent habits can be avoided. For instance, a common parental response to sleep problems in children is to take the child into the parents' bed. However, the potential for the habit of sleeping with the parents to persist is such that identification of a problem early and instituting a more appropriate solution may prevent other difficulties from developing later.

Adolescents attending their family doctor also frequently present with somatic symptoms such as sleep problems or headaches. For a substantial number of these, psychological distress and depression are likely to be present (Angold, 1988). The treatment of adolescent mental health problems is by no means straightforward and depression, in particular, is not as reliably responsive to medication. In recognition of the need for GPs to know how to identify and respond to common problems the Department of Health commissioned a booklet which has been given to all general practices throughout England (Hughes et al, 1994).

\section{Referrals to specialists by GPs}

The 'pathway' to adult psychiatric out-patient and in-patient care has been described by Goldberg \& Huxley (1980). They proposed a model which incorporates filters or gates between one level of care and another. Wolpert \& Fredman (1994) have suggested an adaptation to this model which better suits the pathways for children. As the majority of children with mental health problems are not treated by the specialist services it is of some interest to examine how primary care services select which children and families to refer on. It seems that referral to specialist services is not exclusively determined by the nature of the disorder. An examination of factors within the child showed that increased severity of disturbance, male sex, antisocial symptoms and problems in relationships with peers and teachers (Garralda \& Bailey, 1988) were the most common factors in the child that led to a referral. This is despite the fact that there is a higher percentage of emotional disorders identifiable in general practice. Severity of the defined problem, particularly in the context of family stress, is another key determinant of a referral. In addition the levels of parental anxiety and distress are also significant factors, as is the explicit acknow- 
ledgement by a parent of a desire to be helped (Bailey \& Garralda, 1989).

One service, in response to increased awareness of the significant role played by health visitors, examined the changes in referral patterns which resulted from developing closer relationships with their local health visitors. It led to changes in referrals, with health visitors tackling the straightforward cases more confidently by themselves but referring on those about whom they might previously have felt helpless (Bellenis \& Thompson, 1992). The improved relationship between the two services not only led to more minor difficulties being effectively handled in primary care but also more appropriate referrals to specialist services for the complex cases.

Child mental health services have not always been responsive to the hopes and expectations of parents as to what might result from a referral. Bailey \& Garralda (1989) found that parents expect help to be given directly to the child and hope for explanations about the nature of the child's condition. In addition not all GPs know what happens when children are seen by specialist services and many perceive referral to psychiatrists as stigmatising (Markantonakis \& Mathai, 1990). Bailey \& Garralda also found in their study that most GPs expected child psychiatrists to take over the cases they referred and they did not simply want a diagnosis and advice. This suggests that at present GPs do not feel able to manage these difficulties themselves. Although several studies have indicated that GPs are not fully conversant with the services provided by child mental health services, Mutale (1994) found a surprising amount of agreement between child psychiatrists and GPs about the most effective treatments and treatments of choice. Both rated family therapy and individual counselling higher than other therapeutic interventions.

Both better information being available to GPs and also improved preparation of parents and children are therefore indicated. This, to be effective, clearly depends upon closer relationships

Box 3. Child psychiatric disorders in general practice

Child psychiatric disorders:

- often go unrecognised

- rarely present as pure psychological problems

- often present in the context of somatic symptoms

- are more common among frequent practice attenders developing between primary care and secondary services (Bailey \& Garralda, 1989). Careful discussion with parents about the motives behind a request for help and their expectations should take place before a referral is made. It has been found that congruence between expected and actual recommendations is associated positively with the acceptance of a service.

\section{Adult mental health professionals in primary care}

It is evident that if effective mental health care for children is to be provided within general practice then mental health professionals may have a useful role by working alongside primary care staff or by teaching, training or consultation. Most documented initiatives in this area apply to adult mental health services.

At present in England and Wales there is an uneven distribution of mental health professionals within primary care (Kendrick et al, 1993) but the number of initiatives are increasing. Tyrer (1984) showed that patients prefer to be seen in primary care rather than a hospital setting with the added benefit that continuity of care was facilitated. The effect of setting up psychiatric clinics within primary care led to a $20 \%$ reduction in psychiatric admissions. Interestingly, no extra staff were required within the mental health service as a whole. Patients reported finding the service more convenient and less stigmatising (Ferguson, 1987). A more recent study of setting up a community mental health team in primary care (Jackson et al, 1993), however, did not have the same impact on in-patient admissions but did reduce out-patient referrals. This was associated with a fourfold increase in new referrals of patients with depressive illness but the majority of these were treated in primary care. Hence, more treatable disorders were identified but the burden on secondary care services was lessened.

A study of the impact of specialist social workers working with adult women within primary care also came to interesting conclusions (Corney, 1987). It was women with "acute on chronic" depression who also had marital difficulties that most benefited. They were less depressed at follow-up compared with controls. However, not all groups benefited to the same degree and while assumptions were made that this might reduce behavioural problems in children this was not studied. The key lesson to be learned from this study is that proper evaluation of which groups of patients are likely to 
benefit most is essential and an uncritical allocation of resources may lead to wasted effort.

Darling (1990) demonstrated that liaison between mental health professionals and general practitioners is cost-effective and is of mutual benefit. Twenty-six per cent of the contacts were about patients not in psychiatric care which demonstrates the increased number of patients reached by this approach. Likewise, in another study it was the discussions of assessments, psychological management and communication that were rated as the most important benefits accruing from these closer links (Westbrook \& Hawton, 1991). The debate remains as to how best use such liaison time and whether to concentrate on direct or indirect work. Harris (1994) advocates that a consultant's time in primary care is better spent providing training and support for professional development rather than direct services to patients. Most agree, however, that it is the face to face contact between professionals that makes the difference.

\section{The role of child mental health professionals in primary care}

With reference to child mental health problems Williams (1993) has also called for greater collaboration between primary and secondary practitioners:

"Early contact can result in discussion of treatment plans leading to effective primary care interventions, increased confidence amongst GPs and more rapid treatment of those with serious disorders through better case selection."

Although studies are limited there are some accounts of $\mathrm{CAMH}$ professionals participating in primary care. Subotsky \& Brown (1990) demonstrated that a higher first attendance rate could be obtained by holding a clinic in a general practice setting, and that if a follow-up at a child guidance clinic was required, the attendance rate was very good. A child psychotherapist described the wider influence she had on a primary care team which arose from discussion and thinking about cases rather than the direct work with the child (Sternberg, 1993). Such sessions held in primary care settings are seen as effective means of helping to guide general practice towards an increased understanding of child mental health issues. Thompson \& Place (1995) showed that GPs often have a scanty knowledge of child mental health services and advocated personal contact between
Box 4. Common emotional and behavioural problems that may present to GPs (adapted from Markus et al, 1991)

In early childhood Sleepless nights and nightmares Temper tantrums and breathholding Clinging behaviour

Feeding and bowel problems Bed wetting and soiling

In mid childhood School non-attendance Learning difficulties Disobedience and aggressive behaviour Social withdrawal and shyness

Recurrent abominal pain and headaches

In adolescence

School non-attendance

Delinquency

Substance abuse

Moodiness and irritability

Suicidal thoughts

Social anxieties and withdrawal

Eating problems

primary and secondary services to improve understanding. They showed that holding such clinics promoted child psychiatry referrals. A similar study of the effect of personal contact between a consultant adolescent psychiatrist and GPs (Wells \& Faragher, 1992) showed that referral rates to an adolescent unit were increased.

Within fund-holding practices a recent survey showed that CPNs had more links with practices than did psychologists or psychiatrists (Mutale, 1994). While adult mental health professionals predominated, a significant number of the practices had links with child mental health professionals mainly through regular meetings or practice based clinics rather than through joint assessment activities. There seems little doubt that the increased development of closer contacts is here to stay.

\section{Intervention by members of the primary care team}

Closer contacts should lead to enhanced and more effective child mental health care within general practice. Attitudes are changing and some of the 
fundamental strategies used by child mental health professionals may also be useful to GPs. Markus et al (1991) stress the importance of talking to all relevant family members when making an assessment and discuss the use of family therapy skills amongst other techniques. However, a GP's contact time with each patient is limited so primary care teams are now employing their own workers to undertake mental health work. The development of fundholding has encouraged GPs to provide more services themselves despite the fact that there is no universal agreement about the most effective interventions for treating mental health problems within general practice. One of the most noticeable developments has been that of counselling services though evidence for effectiveness is less than satisfactory. A survey of counsellors in general practices in England and Wales not only demonstrated the uneven distribution but also that more than a third had no specific training in counselling. They dealt with a very broad range of problems, from family and relationship difficulties to substance misuse and psychiatric illness. It is doubtful that counsellors can deal with such a large range of problems (Sibbald et al, 1993).

There is also conflicting evidence about the value of counselling and when it should be used. It has been alleged in the context of working with mentally handicapped children that parent counselling can undermine confidence, lower parental self-esteem and increase dependency on outside agencies (Clement, 1985). However, Davis \& Rushton (1991) in a community setting has demonstrated that "parent advisors", trained with basic counselling skills, can significantly improve many aspects of parental relationships and also benefit the child. There is no doubt that there is a very considerable need for more evaluation in order to be clearer about when and with whom a

Box 5. Child mental health professionals can improve mental health care for children by

Providing more and better information about specialist services

Developing closer relationships with general practitioners

Increasing face to face contact with primary health care workers

Participating in training activites within primary care

Developing interventions suitable for primary care counsellor can be most effective (King, 1994) and brief effective interventions suitable for implementation in primary care need to be developed (Bowman \& Garralda, 1993). Whether the employment of counsellors or increasing the counselling skills of GPs, as advocated by Pringle \& Laverty (1993), is the most effective use of resources, is at present uncertain.

\section{Opportunities for mental health promotion and prevention}

As Offord (1987) pointed out, it is clearly more profitable to prevent developmental, behavioural and management problems than to identify them once established. Numerous opportunities present themselves in general practice for preventive mental health care. The identification and treatment of maternal depression, especially postnatal depression, is particularly important. In addition the anticipation of problems at times of psychosocial transition (e.g. separations, bereavement), sensitive handling of families caring for children with chronic physical illness and the early identification of parent/child relationship problems are other examples of identifiable situations in which preventive action can be taken. Even at the tertiary level of prevention there are opportunities (Hall, 1992). Parents value early diagnosis of permanently disabling disorders for emotional as well as practical reasons. The quality of life may be improved and features such as adaptation, coping ability and family function are facilitated.

Cox (1993) has summarised some of the ways in which preventive action can be taken. He points out that child psychological and psychiatric disorders are not only multifactorial but generated and sustained by a variety of processes. The focus should be the reduction of personal vulnerability, the increase of competence, the reduction of stressors or their impact and the lessening of the adverse effects of particular experiences.

Most of the documented study of the effectiveness of schemes to prevent mental ill health in children have been undertaken by child mental health professionals themselves. While the potential role for GPs is self-evident there has been little in the way of systematic examination of possible strategies. A number of projects with health visitors have been carried out in view of their unique opportunities to have contact with families with young children. Holden et al (1989) 
demonstrated that significant psychiatric improvement in mothers with postnatal depression could be obtained when they were offered counselling by specially trained health visitors. Cox (1993) has summarised some of the other efforts that have been described to train health visitors to alleviate maternal depression, to assist mothers in managing children's behaviour problems or to prevent child abuse. However, much of the evidence for these programmes is equivocal. A persistent problem is that many of the most needy families containing children at the greatest risk are the least cooperative (McGuire et al, 1991). There is relatively little evidence that behaviour of parents can be substantially changed or that changes produced will translate into improved child development or behaviour (McGuire \& Earls, 1991).

Given the high number of children and adolescents with unidentified mental health problems the value of screening perhaps should be considered. Whether this would be cost-effective or whether services would subsequently be available for them is questionable. Nonetheless, in one general practice all adolescents between the ages of 16 and 17 were invited to attend for such an exercise and 50\% did so (Donovan, 1988). The most common problems identified were obesity, depression and acne.

It is early days in the development of evaluated methods of health promotion and primary prevention of mental health disorders in children. Current evidence suggests that single interventions (the 'inoculation' model) are less likely to be effective than continued interventions over time (the 'nutritional' model). However, simple interventions in a single area are unlikely to work on their own (MacFarlane, 1993) and a more comprehensive strategy involving other child care services should probably be considered.

\section{Conclusions}

There is a considerable need for improving the quality and quantity of mental health care for children within general practice settings. Secondary care services should play a part in this process but their resources for direct work should primarily be directed towards those children, young people and families with the most severe or complex disorders. Developments in primary care need to be evaluated thoroughly and research on the effectiveness of interventions should be meticulous to avoid wastage of precious resources.

\section{References}

Angold, A. (1988) Childhood and adolescent depression II: research in clinical populations. British Journal of Psychiatry, 153, 476-492.

Bailey, V., Graham, P., Boniface, D., et al (1978) How much child psychiatry does a general practitioner do? Journal of the Royal College of General Practitioners, 28, 621-626.

Bailey, D. \& Garralda, M. (1989) Referral to child psychiatry: parent and doctor motives and expectations. Journal of Child Psychology and Psychiatry, 30, 449-458.

Bellenis, C. \& Thompson, M. (1992) A joint assessment and treatment service for the under fives. ACPP Newsletter, 14, 262266.

Bowman, F. \& Garralda, M. (1993) Psychiatric morbidity among children who are frequent attenders in general practice. British Journal of General Practice, 43, 6-9.

Clement, J. (1985) Update - Training parents of mentally handicapped children. Newsletter of the Association of Child Psychology and Psychiatry, 7, 2-9.

Corney, R. (1987) Marital problems and treatment outcome in depressed women. British Journal of Psychiatry, 151, 652-659.

Cox, A. (1993) Preventive aspects of child psychiatry. Archives of Disease in Childhood, 68, 691-701.

Darling, C. (1990) Brief encounters in general practice: liaison in general practice psychiatry clinics. Psychiatric Bulletin, 14, 592-594.

Davis, H. \& Rushton, R. (1991) Counselling and supporting parents of children with developmental delay: a research evaluation. Journal of Mental Deficiency Research, 35, 89-112.

Donovan, C. (1988) Is there a place for adolescent screening in general practice? Health Trends, $20,64$.

Evans, S. \& Brown, R. (1993) Perception of need for child psychiatry services among parents and general practitioners. Health Trends, 25, 53-56.

Ferguson, B. (1987) Current comment (vii). Psychiatric clinics in general practice - an asset for primary care. Health Trends, 19, 22.

Garralda, M. \& Bailey, D. (1986) Children with psychiatric disorder in primary care. Journal of Child Psychology and Psychiatry, 27, 611-624.

- \& - (1988) Child and family factors associated with referral to child psychiatrists. British Journal of Psychiatry, 153, 81-89.

Goldberg, D. \& Huxley, P. (1980) Mental Illness in the Community - The Pathway to Psychiatric Care. London: Tavistock.

Gray, D. (1994) Family and marital relationships. In Psychiatry and General Practice Today (eds I. Pullen, G. Wilkinson, A. Wright, et al), pp. 77-92. London: Gaskell.

Hall, D. (1992) Annotation: child health promotion, screening and surveillance. Journal of Child Psychology and Psychiatry, $33,649-657$.

Harris, A. (1994) Specialist outreach clinics. British Medical Journal, 30, 1053.

Holden, J., Sagovsky, R. \& Cox, J. (1989) Counselling in a general practice setting: controlled study of health visitor intervention in treatment of postnatal depression. British Medical Journal, 298, 223-236.

Horne, J. (1992) Annotation: sleep and its disorders in children. Journal of Child Psychology and Psychiatry, 33, 473-487.

Hughes, T., Garralda, M. \& Tylee, A. (1994) Child Mental Health Problems. London: St. Mary's Hospital.

Jackson, G., Gater, R. \& Goldberg, D. (1993) A new community health team based in primary care. Adescription of the service and its effect on service use in the first year. British Journal of Psychiatry, 162, 375-384.

Kendrick, T., Sibbald, B., Addington-Hall, J., et al (1993) Distribution of mental health professionals working on site in English and Welsh general practices. British Medical Journal, 307, 544-546.

King, M. (1994) Counselling services in general practice. The need for evaluation. Psychiatric Bulletin, 18, 65-67.

Kurtz, Z, Thornes, R. \& Wolkind, S. (1994) Services for the Mental 
Health of Children and Young People in England. A National Review. London: Maudsley Hospital and South Thames (West) Regional Health Authority.

MacFarlane, A. (1993) Health promotion and children and teenagers. British Medical Journal, 306, 81.

Markantonakis, A. \& Mathai, J. (1990) An evaluation of general practitioners' knowledge and satisfaction of a local child and family psychiatric service. Psychiatric Bulletin, 14, 328-329.

Markus, A.,, Murray Parkes, C., Tomson, P., et al (1991) Psychological Problems in General Practice. Oxford: Oxford University Press.

McGuire, J. \& Earls, F. (1991) Prevention of psychiatric disorders in early childhood. Journal of Child Psychology and Psychiatry, 32, 129-154.

Mutale, T. (1994) Links between fund-holding general practices and mental health professionals. Psychiatric Bulletin, 18, 603605.

Mutale, T. (1994) Attitudes of general practitioners and child psychiatrists to treatment methods. Psychiatric Bulletin, 18, 668669.

Offord, D. (1987) Prevention of behavioural and emotional disorders in children. Journal of Child Psychology and Psychiatry, 28, 9-20.

Peter, L. (1993) Services for children: primary care. British Medical Journal, 307, 117-120.

Pringle, M. \& Laverty, J. (1993) A counsellor in every practice? British Medical Journal, 306, 2-3.

Rutter, M., Tizard, J. \& Whitmore, K. (1970) Education, Health and Behaviour. London: Longman

Shepherd, M. \& Clare, A. (1981) Psychiatric Illness in General Practice (2nd edn.), Oxford University Press.

Sibbald, B., Addington-Hall, J., Brenneman, D., et al (1993) Counsellors in English and Welsh general practices: their nature and distribution. British Medical Journal, 306, 29-33.

Smeeton, N. (1987) Surveys of mental illness in general practice. Professional Statistician, 6, 8-9.

Smeeton, N., Wilkinson, G., Skuse, D., et al (1992) A longitudinal study of general practitioner consultations for psychiatric disorders in adolescence. Psychological Medicine, 22, 709-715.

Sternberg, J. (1993) "Applied Psychotherapy?" An account of brief work in a medical centre. ACPP Review and Newsletter, 15, 260264

Strathdee, G. \& Williams, P. (1984) A survey of psychiatrists in primary care: the silent growth of a new service. Journal of the Royal College of General Practitioners, 34, 615-618.

Subotsky, F. \& Brown, R. (1990) Working alongside the general practitioner: a child psychiatric clinic in the general practice setting. Child: Care, Health and Development, 16, 189-196.

Thompson, A. \& Place, M. (1995) What influences general practitioners's use of child psychiatry services? Psychiatric Bulletin, 19, 10-12.

Tyrer, P. (1984) Psychiatric clinics in general practice. An extension of community care. British Journal of Psychiatry, 145, 9-14.

Wells, P. \& Faragher, B. (1992) Referral rates to a regional adolescent psychiatric service: a comparison of visited and unvisited general practitioners. Health Trends, 24, 53-56.

Westbrook, D. \& Hawton, K. (1991) Liaison meetings between a psychiatric team and general practitioners: description and evaluation of a pilot project. Psychiatric Bulletin, 15, 328-329.

Williams, R. (1993) Psychiatric morbidity in children and adolescents: a suitable cause for concern. British Journal of General Practice, 43, 3-4.

Wolpert, M. \& Fredman, G. (1994) Modelling the referral pathway to mental health services for children. ACPP Review and Newsletter, 16, 283-288.

\section{Multiple choice questions}

1 Children with psychiatric disorders seen in primary care

a are more likely to be referred to specialist services if female b attend more frequently than those without psychiatric disorders

c usually present with an emotional problem as the main complaint

d are registered with their fathers more than their mothers

e have been shown to best respond to direct counselling

2 Which of the following statements about general practitioners are true

a they spend only $5 \%$ of their time working with children

b on average they appear to have a poor understanding of child and adolescent mental health services

c they have more direct contact with adult mental health services than child mental health services

d they expect to see adolescents more frequently than younger children

e they record psychiatric diagnoses in younger children more frequently than adolescence

3 Evidence exists to show that

a counselling services within primary care are well targeted

b parental anxiety is associated with referral of children to specialist services

c parents often consult health visitors first about problems in young children

d counselling of parents will prevent adolescents developing psychiatric problems.

e the majority of children with identifiable psychiatric disorder are not referred to specialist services.

MCQ answers

123

a F a F a F

b $T$ b $T$ b $T$

c $\mathrm{F} \quad \mathrm{c} T \quad$ c $\mathrm{T}$

d F d F d F

e $\begin{array}{lllll}F & \text { e } & F & \text { e } & T\end{array}$ 